\title{
Full field apparent resistivity definition of borehole TEM with grounded electric source
}

\author{
Junjie Wu, Qingquan Zhi, Xiaohong Deng \\ Institute of Geophysical and Geochemical Exploration \\ Chinese Academy of Geological Science \\ Langfang, China
}

\begin{abstract}
Grounded electric source Borehole TEM is introduced in this paper to find the deep conductive ore body, particularly in areas where the ability of surface TEM to define a target is limited either by large depths or by interfering conductive bodies such as overburden shallow sulphides and peripheral mineralized horizons. In this method the grounded electric transmit system is deployed on the surface and receive the three-component TEM response along borehole. As receiver is nearer to ore body through the borehole than on surface, more reliable TEM response of ore bodies can be obtained. Researches show that the detection depth of grounded electric source system is deeper and more suitable for complex terrain area deep prospecting than magnetic source system. Considering there is no conventional explanation method for the grounded electric source borehole TEM, global apparent resistivity is introduced in this paper to provide a reliable explanation method. Global apparent resistivity is defined by the inverse function relationship between induction magnetic field and resistivity of half space. Numerical model calculation results show that underground information can be showed reliably by global apparent resistivity curves along borehole. The application of this study can provide strong support for the deep prospecting in mining area.
\end{abstract}

Keywords-grounded electric source;borehole TEM; threecomponent response; global apparent resistivity; deep exploration

\section{INTRODUCTION}

Surface-to-borehole transient electromagnetic (TEM) method can be useful in detecting subsurface fracture zones and mineral deposits which are nearby, but not intersected by boreholes. In this method a transmitting source is deployed on the surface while a receiving coil is moved down a borehole in order to detect conductive bodies in the vicinity of the borehole. As the noise level is lower in borehole than surface, and the probe in borehole is closer to deep targets, useful geological information within the range of several hundred meters around the borehole can be obtain in borehole, which can raise the ore prospecting effect. The advantages of borehole TEM method will be more prominent, particularly in areas where the ability of surface TEM to define a target is limited either by large depths or interfering conductive bodies

Author: Wu Jun-jie, Institute of Geophysical and Geochemical Exploration, Chinese Academy of Geological Science, Langfang, China,.E-mail: wujunjie@igge.cn

Fund: Basal research fund of central public-interest scientific institution (AS2015J09)

\author{
Junjie Wu, Xiu Li, Jianlei Guo \\ School of Geology Engineering and Geomatics \\ Chang'an University \\ Xi'an,China
}

such as overburden, shallow sulphides and peripheral mineralized horizons ${ }^{[1]}$.The transmitting source of TEM method can be divided into magnetic source and electric source. The magnetic source is loop source and electric source is grounded source. There are many studies on Surface-toborehole TEM method with magnetic source ${ }^{[2]}$. At present, some progress has been made in the three-dimensional forward modeling, data processing and interpretation of field borehole data ${ }^{[4-7,10]}$.

However, sometimes it is hard to carry out surface-toborehole TEM with loop source in the complex mountainous terrain. Researches show that the electric source TEM method has great potential in deep exploration ${ }^{[8]}$.

The surface-to-borehole TEM method with grounded electric source configuration introduced in this paper is transmitting with grounded electric source and receiving threecomponent TEM response along borehole with receiver probe. For existing holes, the transmitting source can be selected to lay out in a wider range (around a few kilometers) to avoid hard terrain with electric source, and exploration of boreholes around the area can also be carried out with the same transmitting source, which largely reduce construction difficulty and improve work efficiency. This paper aims to obtain more intuitive and reliable preliminary interpretation method. Full field apparent resistivity interpretation method is introduced to provide technical support in hard terrain conditions. Its results can provide reference information for the follow-up inversion interpretation.

TEM response of electric dipole in a homogeneous half space in underground medium is given in this paper, and the TEM response of electric source in borehole can be obtained by the way of electric dipole superposition. The relationship of magnetic field in the underground produced by electric source and the resistivity is monotonous in a certain range. Therefore, full field apparent resistivity can be defined with the magnetic field, using inverse function theorem. Theoretical model computation results show that geological information can be obtained reliably with the full field apparent resistivity method, which provides an effective approach for the interpretation method of grounded electric source borehole TEM method.

\section{Full Field Apparent Resistivity Definition}

TEM response underground of galvanic source can be obtained by the way of electric dipole superposition (Kerry 
Key, 2009). Calculation result shows that three-component magnetic field curves in the borehole are all monotonic functions of resistivity, and full field apparent resistivity is adopted in this paper.

With surface-to-borehole configuration of electric source, the TEM response in borehole can be expressed as

$$
B=f\left(x, y, z, \rho, a, I_{0}\right)
$$

Where $\boldsymbol{x}, \boldsymbol{y}, \boldsymbol{z}$ are the coordinates respectively and $\boldsymbol{t}$ is the sampling time channel, $\boldsymbol{\rho}$ is the resistivity, $\boldsymbol{I}_{\boldsymbol{0}}$ is the current, $\boldsymbol{a}$ is the geometrical parameters of transmit source, $\boldsymbol{B}$ represents the observed field value function of the configuration parameters and model parameters.

The configuration parameters and observation parameters can be seemed as constant parameters of the function when the configuration has been fixed, these parameters can be omitted, that is

$$
\begin{gathered}
B=f(\rho) \\
\text { As } g(\rho)=\left[\frac{B-f(\rho)}{B}\right]^{2} \text {, make Taylor expansion of }
\end{gathered}
$$

$g(\rho)$ at $\rho_{0}$ point, ignore the higher order terms of the two order, and transposition terms, iterative format can be constructed as

$$
\rho=\rho_{0}+\frac{g(\rho)-g\left(\rho_{0}\right)}{g^{\prime}\left(\rho_{0}\right)}
$$

Eq. (3) is the basic iterative format for the iterative calculation of the apparent resistivity value. As the method is independent of parameters of coordinates in space and time during the calculation of apparent resistivity, this definition belongs to full field method.

\section{ReSUltS AND ANALYSIS}

In order to prove the correctness of the expression of global apparent resistivity definition method, series onedimensional model were computed. Considering layout restrictions, this paper only show the results of $\mathrm{D}$ type model $\left(\rho_{1}=200 \Omega \cdot \mathrm{m}, \quad \rho_{2}=10 \Omega \cdot \mathrm{m}, \mathrm{h}_{1}=300 \mathrm{~m}\right)$ and $\mathrm{H}$ type model $\left(\rho_{1}=200 \Omega \cdot \mathrm{m}, \rho_{2}=10 \Omega \cdot \mathrm{m}, \rho_{3}=200 \Omega \cdot \mathrm{m}, \mathrm{h} 1=200 \mathrm{~m}, \mathrm{~h} 2=50 \mathrm{~m}\right)$.

Fig. 1 shows the calculation results of D type model, and Fig. (1a) shows Z-component decay curves in borehole with different depths. Fig. (1b) shows TEM response plot curves with different time channels. In the Fig. (1b), $0.1 \mathrm{~ms}$ and $0.125 \mathrm{~ms}$ curves shows no significant anomalies in the early stage, and there is a significant anomaly at the $300 \mathrm{~m}$ depth from $0.199 \mathrm{~ms}$ to $1.99 \mathrm{~ms}$. The response amplitude gradually increased with the depth and became smaller after reaching the maximum. The curve shows no significant abnormality after $5.01 \mathrm{~ms}$.

Fig. (1c) shows apparent resistivity curves at different depths. It can be seen that the first segment of apparent resistivity curve of the station on the surface is close to the resistivity of the first layer as $200 \Omega \cdot \mathrm{m}$ value, and decline until close to the resistivity of the second layer value as $10 \Omega \cdot \mathrm{m}$, which shows a typical D type characteristic. And with the increasing of the depth, the first segment of apparent resistivity curve shows more and more obscure, and decreases with time till reach at the second layer resistivity values. Although the first segment apparent resistivity curves at early stage at the depth of $360 \sim 500$ meters is dozens of $\Omega \cdot \mathrm{m}$, the curves maintained a high trend curve to low. Analysis of this phenomenon, we can get that the electrical information reflected by different depth apparent resistivity values is different.

The apparent resistivity curve of the station on the ground completely reflects the section information from the surface to the underground, where the curve is complete, and the section is completely consistent with the section. And the apparent resistivity curve of the underground station mainly reflect the resistivity distribution around a certain range. Especially when the measuring station is in the low resistivity layer, the apparent resistivity curve is mainly reflect the low resistivity layer, the apparent resistivity value will be low. But this difference is appear only in the early stage, the difference will became smaller with time increasing.

In this paper, so as to reflect the apparent resistivity change of underground media along the borehole, the apparent resistivity curves of same time channel are plotted into a group. The apparent resistivity curves with different depth at $0.199 \mathrm{~ms} \sim 3.16 \mathrm{~ms}$ and the models are shown in Fig. (1d). It can be seen from the fig., the apparent resistivity of $0.199 \mathrm{~ms}$ at the depth from 0 to $100 \mathrm{~m}$ is close to $200 \Omega \cdot \mathrm{m}$, and become smaller gradually with depth, and ultimately maintained at about $60 \Omega \cdot \mathrm{m}$. This curve is typical D curve, which consistent with the model. It can be seen that with the increase of time D type feature is less obvious, especially at $3.16 \mathrm{~ms}$. This phenomenon is consistent with the previous analysis.

Calculation results of $\mathrm{H}$ type model are shown in Fig. 2. It can be seen from the Fig. (2a), the early stage TEM response curves gradually become flat with the depth increasing, which is caused by the slow change of the early magnetic field, and it is similar to that of the D type curve. From the TEM response profile curve in Fig. (2b), we can see that there is no abnormal display in the early stage, with the increase of the time channel, the abnormal amplitude becomes smaller. All the apparent resistivity curve at different depth can show the characteristics of " $\mathrm{H}$ " type model, but the first segment apparent resistivity curves are different. The reason has been analyzed in the previous paper, which is due to the different range of different apparent resistivity values. When the depth of the measure station is increased and gradually close to the low resistivity layer, the apparent resistivity value of the early stage response will be lower, and its influence on the later stage is relatively small. The low resistivity anomaly reflected in Fig. (2d) is basically consistent with the theoretical model. It can be seen that the early stage TEM response of the measuring station near the low resistivity layer will show a low value of the apparent resistivity. When the station is located below the low resistivity layer, the influence of the low resistivity layer still exists, making the early stage apparent resistivity curve lower than value due it. 


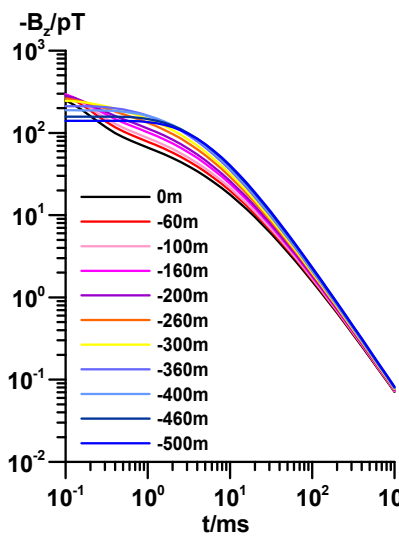

(a)

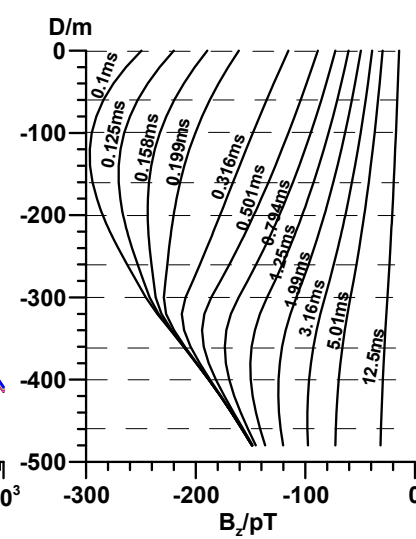

(b)

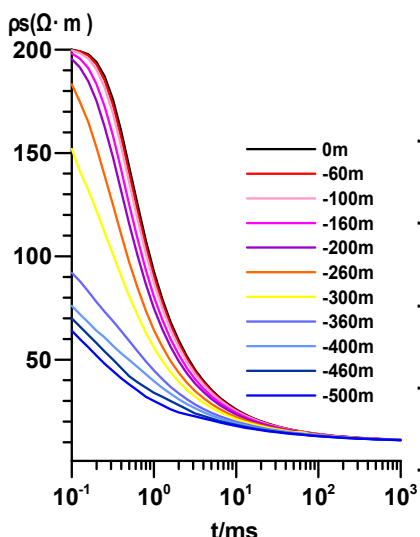

(c)

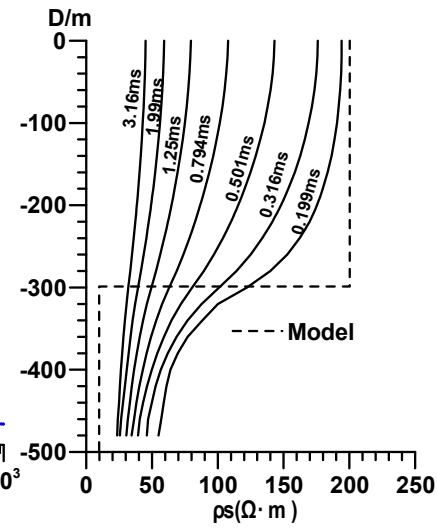

(d)

Fig. 1 Calculation results of $\mathrm{D}$ type modela. $Z$ component TEM decay curves $\quad$ b. $Z$ component TEM response along hole c. Apparent resistivity curves of different depth d. Apparent resistivity along hole

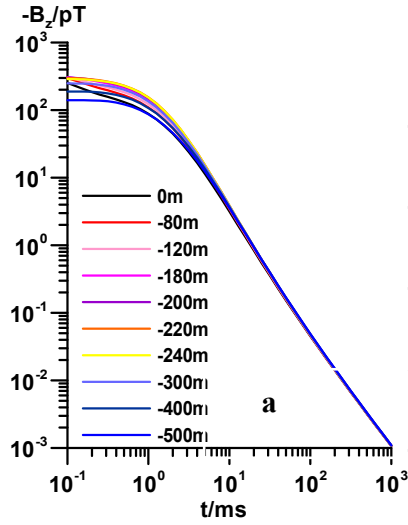

(a)

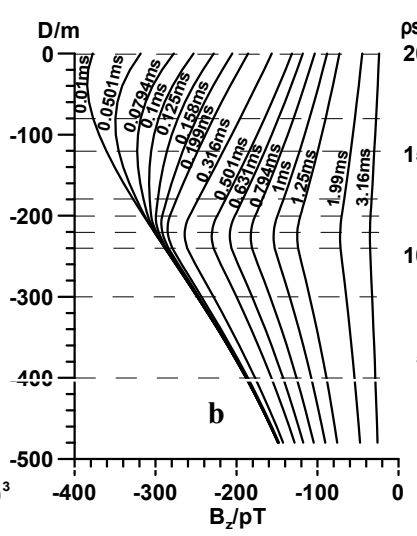

(b)

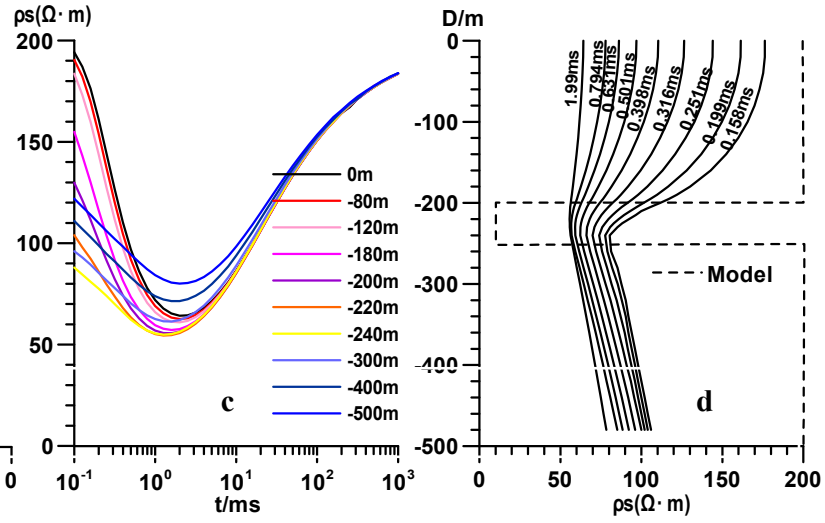

(c)

(d)

Fig. 2 Calculation results of $\mathrm{H}$ type model a. Z component TEM decay curves b. Z component TEM response along hole c. Apparent resistivity curves of different depth d. Apparent resistivity along hole

\section{CONCLUSIONS}

- The full field apparent resistivity definition method of surface-to-borehole TEM with grounded electric source based on the inverse function is introduced in this paper, and theoretical calculation results of the model show that the global apparent resistivity curve can reliably reflect the underground electrical distribution along the borehole.

- Calculation results indicate that the apparent resistivity curves of stations on surface are different with the curves of underground stations. The apparent resistivity curve on surface reflect the electrical distribution from the surface to a certain depth underground, while the apparent resistivity value in borehole mainly reflect the electric distribution around the station rather than reflect the overall geoelectric section. This difference is very obvious in the apparent resistivity curve at the early stage, and the apparent resistivity curve become consistent with the surface curve at the late stage. The apparent resistivity of early stage with different depth can reflect the change of electric property in depth.

\section{REFERENCES}

[1] Tom Eadie, Guido Staltari.1987.Introduction to down hole electromagnetic methods. Exploration Geophysics:247 351.

[2] C T Barnett.1984. Simple inversion of time-domain electromagnetic data.Geophysics, 49(7):925 933.

[3] A C Duncan.1987.Interpretation of down-hole transient EM data using current filaments.5th ASEG conferenc:36 39.

[4] N A Hughes.1996. Three Component DHEM surveying at Balcooma.Exploration Geophysics, 27:77 89.

[5] J P Cull.1993. Downhole Three Component TEM Probes.Exploration Geophysics,24:437 442.

[6] Z Zhang,J Xiao.2001.Inversions of surface and borehole data from large-loop transient electromagnetic system over a 1-D earth.Geophysics,66(4):1090 1096.

[7] H J Kim,K H Lee,M Wilt,2003. A fast inversion method for interpretating borehole electromagnetic data.Earth Planets Space,55:249 254. 
[8] Xue G Q, Chen W Y, Zhou N N, et al.2013.Short-offset TEM technique with a grounded wire source for deep sounding. Chinese J. Geophys (in Chinese), 56(1):255-261.

[9] Li X, Zhang Y Y, Lu X S, et al.2015.Inversion Synthetic Aperture Imaging of Ground-Airborne transient electromagnetic method with a galvanic source. Chinese J. Geophys(in Chinese), 58(1):277-288.
[10] Kerry Key.2009.1D inversion of multicomponent, multifrequency marine CSEM data: Methodology and synthetic studies for resolving thin resistive layers. GEOPHYSICS, 74(2):F9 F20. 